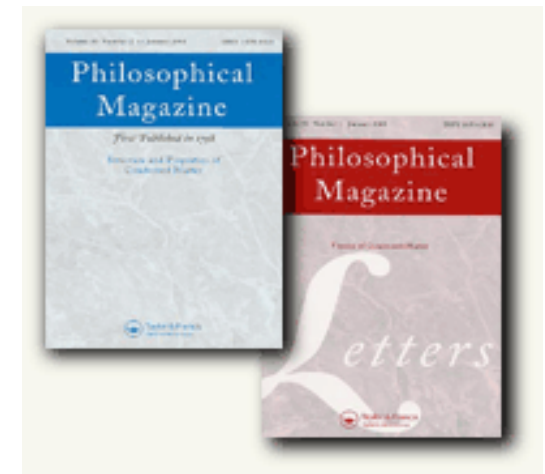

\title{
Structure of liquid bismuth calculated from pseudo- potentials and molecular dynamics
}

\begin{tabular}{|r|l|}
\hline Journal: & Philosophical Magazine \& Philosophical Magazine Letters \\
\hline Manuscript ID: & TPHM-09-Aug-0362.R1 \\
\hline Journal Selection: & Philosophical Magazine \\
\hline Author: & 02-Oct-2009 \\
\hline Complete List of Authors: & $\begin{array}{l}\text { Grosdidier, Benoit; Université Paul Verlaine - Metz, , Institut de } \\
\text { Chimie, Physique et Matériaux } \\
\text { Es Sbihi, Driss; Université Paul Verlaine - Metz, Laboratoire de } \\
\text { Physique des Milieux Denses, Institut de Chimie, Physique et } \\
\text { Matériaux } \\
\text { Ben Abdellah, Abdellatif; Université Abdelmalek Essaadi, Faculté } \\
\text { des Sciences et Techniques de Tanger } \\
\text { Gasser, Jean-Georges; Université Paul Verlaine - Metz, LPMD; } \\
\text { (personnal) }\end{array}$ \\
\hline Keywords (user supplied): & bismuth, pseudo potential \\
\hline & \multicolumn{2}{|l}{} \\
\hline Keywords: & atomic structure, liquid metals, molecular dynamic simulations \\
\hline
\end{tabular}

\section{(5) ScholarONE \\ Manuscript Central}




\title{
Structure of liquid bismuth calculated from pseudo-potentials
} and molecular dynamics

\author{
D. Es Sbihi ${ }^{1}$, B. Grosdidier ${ }^{1,1}$, A. Ben Abdellah ${ }^{1,2}$ and J. G. Gasser ${ }^{1}$ \\ ${ }^{1}$ Laboratoire de Physique des Milieux Denses, Institut de Chimie, Physique et Matériaux \\ Université Paul Verlaine - Metz \\ 1, Bd D. F. Arago 57078 Metz Cedex 3 France \\ ${ }^{2}$ Groupe de Recherche de Physique-Mécanique, Université Abdelmalek Essaadi, \\ Faculté des Sciences et Techniques de Tanger B.P. 416 Tanger Maroc
}

\begin{abstract}
Earlier works on liquid bismuth have been done by using 5 conduction electrons. However this metal presents, in the liquid state, a gap in its electronic density of states which separates clearly the $\mathrm{s}$ and the $\mathrm{p}$ bands. Thus the number of free electrons to be considered is affected by the existence of the gap. Earlier calculations on the structure of liquid bismuth with five conduction electrons did not give satisfactory results. We calculated it by Molecular Dynamics using an effective potential derived from the Empty Core Potential (ECP) and from the local Optimised Model Potential (OMP) and compared the results obtained with 3 (p) and $5(s+p)$ conduction electrons. Our results, obtained for the first time to our knowledge for liquid bismuth at different temperatures, with 3 conduction electrons and OMP is in very good agreement with the Waseda structural experimental data. This approach using 3 conduction electrons is confirmed by resistivity calculation using t-matrix model.
\end{abstract}

Key Words: liquid metal, bismuth, atomic structure, pseudo-potentials, molecular dynamics.

\footnotetext{
${ }^{1}$ author for correspondence: e-mail grosdidi@ univ-metz.fr Phone: 33 (3) 873158 80, fax : 33 (3) 87315801
} 


\section{Introduction}

The bismuth-lead alloy is used as coolant in nuclear reactors [1]. The knowledge of the physical properties of this heavy metal is thus essential for this technology [2,3]. In this paper we propose a new approach, never used to our knowledge, which allows modelling correctly the structure of liquid bismuth. The structure factor has been calculated by several authors [4,5] who have some difficulties to explain simultaneously the position and the amplitude of the first peak and the large q scattering vector oscillatory behaviour. The pseudo-potential formalism [6-9] for bismuth has been used by Hafner [5]. Their results, regarding the peaks positions, are quite satisfactory but discrepancies appear on their amplitudes. We used two pseudo-potentials. The local Ashcroft Empty Core Potential (ECP) [8] even used by Hafner and Heine [10] and the local Optimized Model Potential (OMP) proposed by Shaw [9], but never used to our knowledge for structure calculations. The form factors and the effective potentials are calculated with the Ichimaru-Utsumi [11] dielectric screening function. With these ECP and OMP local pseudo-potentials it is necessary to choose the relevant parameter. Often these parameters are fitted from the experimental data such as the electrical resistivity. This choice is not the best one because the resistivity is a scalar and is written as an integral in the Ziman formula [12]. Several compensations may appear in this integral. The fit of the parameters on the experimental structure is more pertinent; indeed the agreement of the two functions must be achieved in a large range. We wished to consider the Density Of States (DOS) data measured by Indlekofer [13] and those calculated by Hafner and Jank [5] which are displayed in figure 1. They show that there is a wide gap between the $\mathrm{s}$ and the $\mathrm{p}$ bands. In other words the valence 5 for bismuth must be replaced by valence 3 which is physically coherent with DOS data. On the other hand, according to Tamaki et al. [14], the effective ionic radius of $\mathrm{Bi}$ ion in liquid $\mathrm{Bi}-\mathrm{Zn}$ alloys is

estimated to be $1.23 \AA$ which is very close to that of $\mathrm{Bi}^{3+}$ ion $(1.20 \AA)$ and very different from that of $\mathrm{Bi}^{5+}$ ion $(0.74 \AA)$. The best agreement is obtained on the transport electronic properties of liquid $\mathrm{Cu}-\mathrm{Bi}$ alloys by Khalouk et al. using three conduction electrons [15].

We took into account these experimental results in order to carry out our structure calculations. To our knowledge, there is no pseudo-potential of which the parameters for bismuth have been calculated with valence 3. We have been led to determine ourselves these 


\section{Theory and theoretical tools}

\section{A. Effective potential}

The local pseudo-potential theory and the definition of the ingredients are given in the references [6-9]. The expression of the ion-ion effective potential $V(r)$ calculated from the pseudo-potential is given by:

$$
V(r)=\frac{Z^{2}}{r}\left(1-\frac{2}{\pi} \int_{0}^{\infty} F^{N}(q) \frac{\sin (q r)}{q} d q\right),
$$

where $Z$ is the chemical valence. The quantity $r$ is the inter-ionic distance. The normalized energy wave number characteristic $F^{N}(q)$ in reciprocal space, is defined from the Fourier transform of the local electron-ion model potential $w_{0}(q)$ :

$$
F^{N}(q)=\left(\frac{\Omega_{0} q^{2}}{4 \pi Z}\right)^{2} w_{0}(q)^{2} \frac{1}{1-G(q)}\left(1-\frac{1}{\varepsilon(q)}\right),
$$


where $\Omega_{0}$ is the mean atomic volume. The quantity $\varepsilon(q)$ is the dielectric screening function, and the function $G(q)$ is the local field correction. This correction becomes more important when the electronic density increases. A lot of expressions for the local field correction have been developed in the literature. In this work, we shall choose the Ichimaru-Utsumi [11] (IU) expression considered as one of the best. We use the local pseudo-potential, whose expression in the real space is:

$$
w_{0}(r)=\left\{\begin{array}{cc}
A & r<R_{C} \\
-\frac{Z}{r} & r<R_{C}
\end{array},\right.
$$

where $R_{C}$ is the core radius whose value can be fitted on physical properties such as the resistivity [17]. The parameter $A$ is equal to zero for the Ashcroft [6] Empty Core Potential (ECP) or is related to the core radius $R_{C}$ by the optimization condition, for the local Shaw [9] Optimized Model Potential (OMP) $\left(A=-Z e^{2} / R_{C}\right)$. The Fourier transform $w_{0}(q)$ of $w_{0}(r)$ is expressed as:

$$
\begin{array}{ll}
w_{0}(q)=-\frac{4 \pi Z}{\Omega_{0} q^{2}} \cos \left(q R_{C}\right)=w_{0}^{E C P}(q) & (\mathrm{ECP}), \\
w_{0}(q)=-\frac{4 \pi Z}{\Omega_{0} q^{2}} \frac{\sin \left(q R_{C}\right)}{q R_{C}}=w_{0}^{O M P}(q) & (\mathrm{OMP}) .
\end{array}
$$

In order to have the same node of the $w_{0}^{E C P}(q)$ and $w_{0}^{O M P}(q)$ form factors, the condition $R_{C}^{O M P}=2 R_{C}^{E C P}$ must be achieved. Equations (1), (2), (3) (4) and (5) show that the chemical valence $Z$ plays a determinant role in the expression of the effective potential.

\section{B. Molecular dynamics}

The structure is calculated by a simulation code. This code uses the standard Verlet [18] algorithm and processes in NVT ensemble. The number of involved particles in the cubic simulation box is equal to 4000 . The side length is equal to $35 \AA$. The time step $\delta$ t is equal to $7 \cdot 10^{-15} \mathrm{~s}$ and the number of iterations after thermalisation is equal to 40000 . The code computes the pair correlation function $g(r)$ for each configuration and gives the its mean value. The static structure factor $S(q)$ is simply obtained from the pair correlation function $g(r)$ by Fourier transform. 


\section{Results and discussion}

For each run temperature the bismuth density is calculated using Lucas [19] compilation of density as a function of temperature under the form: $d=a-b\left(T-T_{M}\right)$ where $T_{M}$ is the melting temperature of the metal. The calculated density values for the three temperatures 300,550 and $800^{\circ} \mathrm{C}$ are respectively $0.02890,0.02795$ and $0.02700\left(\right.$ atom $\left./ \AA^{3}\right)$.

We first calculate the four effective potentials for liquid bismuth, for both values $(Z=3$ and $Z=5$ ) of the number of conduction electrons using ECP and OMP local pseudo-potentials. The same node $q_{0}$ of the form factor has been used for ECP and OMP for each valence. This induces the relation $R_{O M P}=2 R_{E C P}$. The effective potentials are presented in figure 2 . We observe that with 5 conduction electrons the effective potentials obtained from ECP and OMP are attractive and oscillate normally. Those calculated with ECP are deeper than those calculated with OMP. When we use the valence 3, the position of the hard part of the effective potentials decreases and a shoulder (OMP) or a pre well (ECP) appears. For the potential obtained by ECP the pre well is deeper than $k_{B} T$. We shall see that it leads to the system solidification. We calculated the bismuth structure using ECP pseudo-potential and valence 5 for both values of the core radius $R_{E C P}=0.529 \AA$ and $R_{E C P}=1.005 \AA$, and with valence 3 , taking into account the existence of the gap, for both values of the core radius $R_{E C P}=0.587 \AA$ and $R_{E C P}=0.878 \AA$ The various effective potentials (figure 3 ) and the corresponding pair correlation functions (figure 4) are presented. These values of the core radius have been chosen because both of them represent approximately the same height of the first peak of the experimental pair correlation function while the others give the same position of the first peak. Figure 3 shows in the case of valence 5 that the effective potential calculated for $R_{E C P}=1.005 \AA$ exhibit a well of which the energy depth is much higher than $k_{B} T$; the corresponding pair correlation functions (figure 4) is tending to a solid like. The effective potential calculated with $R_{E C P}=0.529 \AA$ (figure 3) presents a shoulder located at $3.13 \AA$. The minimum depth is comparable to $k_{B} T$ and its position is pushed to a $r$ value near $4.38 \AA$. Two peaks with nearly the same height appear in its corresponding pair correlation function, the first at $2.75 \AA$ near the shoulder of the potential at $3.13 \AA$, the second at $4.12 \AA$ near the first minimum of the potential at $4.38 \AA$. Figure 4 show well that, with valence 5 , it 
is impossible to fit the radius parameter $R_{E C P}$, and to be reasonably near the experimental data. As soon as we adjust the position we lose on the amplitude of the peaks on the pair correlation. In the case of valence 3, the same figures 3 and 4 show that we are not able to reproduce the experimental structure of liquid bismuth. The potential leads to solidification as shown in figure 4 for the value of the core radius $R_{E C P}=0.878 \AA$. For $R_{E C P}=0.587 \AA$, the pair correlation function is liquid like. The position of the first peak is located at $2.75 \AA$, the experimental one is at $3.40 \AA$. We concluded that it is impossible to reproduce the structure of liquid bismuth from ECP pseudo-potential for the two values 5 and 3 of the valence.

We now use the local Shaw potential which has the advantage of having no discontinuity. We first present our results with valence 5. Figure 5 shows the effective potentials calculated for various values of Shaw core radius $R_{O M P}(0.95 \AA, 1.00 \AA$ and $1.05 \AA)$. One observes that for $R_{O M P}=1.00 \AA$ and $1.05 \AA$, the deepest minimum is located directly after the hard part, whereas for $R_{O M P}=0.95 \AA$ this minimum is shifted after the first oscillation of the potential. The minimum is much lower than $k_{B} T$. This result hinders the possibility of adjusting the amplitudes of the calculated main peaks on the experimental curves. This appears clearly, on the pair correlation functions (figure 6) and on the corresponding structure factors (figure 7). The OMP potential with valence 5 allows obtaining a satisfactory result on the different peaks positions. The amplitudes of the main peaks of the pair correlation functions are lower than the experimental ones, while the structure factors are higher than the experimental one. With $R_{O M P}=1.05 \AA$ we obtain nearly the same height of the calculated and experimental pair correlation functions main peaks (2.45 instead of 2.55). Nevertheless the first calculated minimum is lower ( 0.63$)$ than the experimental one $(0.80)$. The oscillations are in phase. The first maximum value of the experimental structure factor curve is 2.0 ; our calculation gives 2.6 while Hafner [5] obtains 2.9. The oscillations of the structures factor are in phase, the best result being obtained with $R_{O M P}=1.05 \AA$.

We present in figure 8.a the effective potential of bismuth at $300{ }^{\circ} \mathrm{C}$ obtained with $R_{O M P}=1.174 \AA$ using the OMP and 3 conduction electrons. Its shape is similar to those achieved by Dzugutov-Dahlborg [4] and Hafner-Jank [5]. We observe a shoulder on the effective potential, which characterises the repulsive nature, checked by the low experimental 
It is an important improvement on earlier calculations which proves that using $Z=3$ for liquid bismuth gives a very good pair correlation function and structure factor. We performed the same calculations for liquid bismuth at $550{ }^{\circ} \mathrm{C}$ and $800{ }^{\circ} \mathrm{C}$ using the same assumptions (OMP and valence three). The results are presented respectively in figure 8.b, 8.c, 9.b, 9.c and 10.b, 10.c.

In order to complete this study, we determined the characteristic parameters of respectively $g(r)$ and $S(q)$ for the three temperatures. All values are given in Tables 1 and 2. Here it is important to refer to the criterion of the classification of the structure of liquid metals due to Waseda [16]. According to this criterion the structure of liquid metals may be classified into three categories, "categories I, II and III". The liquid structure of "category I" such as liquid lead is characterized by the symmetrical first peak of $S(q)$ and also by the fact that the ratio $q_{1} / q_{2}$ is 1.86 where $q_{1}$ and $q_{2}$ are, respectively, the positions of the first and second peak of $S(q)$. Waseda [16] showed that the structure of this "category I"" can be well reproduced by the hard sphere structure factor. Also, this ratio, 1.86, keeps its value even at a low packing fraction, $\eta$, or at a high temperature. Therefore, the liquid structure of "category I' is considered to be rather simple. The asymmetrical first peak is observed for the $S(q)$ of “category II" liquid metals such as liquids $(\mathrm{Zn}, \mathrm{Cd}, \mathrm{Hg})$. A shoulder appears on the rigth side of the first peak of the $S(q)$ for "category III" liquid metals. Liquid bismuth can be classified into this "category III". The ratio $q_{1} / q_{2}$ of both "categories II and III" differs considerably from 1.86. The temperature dependence of this ratio has not yet been investigated for "categorie III", and especially for bismuth. Therefore, the temperature dependence of this ratio, $q_{1} / q_{2}$, was investigated in detail for this liquid metal at three temperature. This ratio is found to remain close to 1.86 even at higher temperatures than the 
melting temperature, as can be seen in (Table I). This indicates that the deviation from the simple liquid metals is significant for liquid bismuth even at a high temperature.

The characteristic parameters of these $g(r)$ 's are given in Table II, in which the first coordination numbers are also shown. The first coordination numbers, which represent the numbers of atoms around the first peak of $g(r)$, were calculated from the fourth method of Waseda's book [16]. The height of the first peak in $g(r)$ becomes lower with the increase of the temperature for liquid bismuth, as is the case of many other liquid metals. The characteristic behaviour of the pair correlation function $g(r)$ for liquid bismuth appears between the first and second peak positions located at $r_{1}$ and $r_{2}$. In this range $\left[r_{1}, r_{2}\right]$, this function $g(r)$ exhibits a flat shape without deep minimum. Its behaviour is different of that of "category I'" liquid metals which present clear minimum, such as liquid aluminium or lead... The first coordination number of liquid bismuth decreases monotonously from 9.47 to 8.21 with the increase of temperature from $300{ }^{\circ} \mathrm{C}$ to $800{ }^{\circ} \mathrm{C}$, as explicitly shown in Table II.

The choice of the number of $p$ electrons as the number of conduction electrons considerably improves structure factor calculations. These values are consistent with the presence of the gap of the experimental DOS [13]. We check our choice by calculating it electrical resistivity based on the extended Ziman formalism [21]. We start by constructing the muffin potentials necessary to the electrons scattering. The muffin tin potentials building following the method of Mukhopadhyay [22] is explained in reference [23]. We calculate the resistivity for liquid bismuth at 300,550 and $800^{\circ} \mathrm{C}$ with the two sets of valencies 5 and 3 . All calculations are performed in the frame of the Esposito approach [24] and are clearly explained in the reference [23]. Our results are presented in Table 3 together with experimental values [15] (second column). It appears clearly that, the values calculated with only $\mathrm{p}$ conduction electrons are always closer to the experimental resistivities. In every case our assumption improves the resistivity calculation of a factor nearly equal to 2.5 .

\section{Conclusion}

Calculations of properties of bismuth liquid metals with the Shaw local pseudo-potentials three conduction electrons as valence give good results. The results are improved and better than those obtained earlier by using other local pseudo-potentials [3-4]. This new approach using the local OMP may serve for the study of other properties such as the dynamical 
structure factor ${ }^{\circ}[27]$. These assumptions may also be used to calculate the structure of bismuth-lead liquid alloy which had an increasing interest as coolant and spallation source in the conversion of radioactive element in accelerator-driven systems (ADS) or for nuclear reactor cooling.

\section{References}

[1] B.F. Gromov et al., Nucl. Eng. Des. 173 (1997) p.207.

[2] I Kaban, W Hoyer, Yu Plevachuk and V Sklyarchuk, Phys.: Condens. Matter (2004) p.6335.

[3] F. Sar, S. Mhiaoui and J.G. Gasser, J. Non-Cryst. Solids 353 (2007) p.3622.

[4] A. M. Dzugutov, U. Dahlborg, Phys. Rev. A 40 (1989) p.4103.

[5] J. Hafner and W. Jank, Phys. Rev. B 45, (1992) p.2739.

[6] W. A. Harrison, "Pseudopotentials in the Theory of Metals", Benjamin, New York (1966).

[7] A. O. E. Animalu, Phil. Mag. 11 (1965) p.379.

[8] N. W. Ashcroft, Phys. Let. 23 (1966) p.48.

[9] R. W. Shaw, Phys. Rev. B 5 (1972) p.4742.

[10] J. Hafner and V. Heine, J. Phys: Met. Phys. 13 (1983) p.2479.

[11] S. Ichimaru and K. Utsumi, Phys. Rev. B 24 (1981) p.7385.

[12] J.M. Ziman, Phil. Mag. 6, (1961) p.1013.

[13] G. Indlekofer, A. Pflugi, P. Oelhafen; J. Non-Cryst. Solids 117/118 (1989) p.351.

[14] S. Tamaki, S. Takeda, S. Harada, Y. Waseda, and E. Matsubara, J. Phys. Sot-Japan, 55, (1979) p.4296.

[15] K. Khalouk, C. Chaib and J. G. Gasser, Phil. Mag. 89 (2009) p.249.

[16] Y. Waseda Y, “The Structure of Non-Crystalline Materials”, McGraw-Hill, New York (1980).

[17] A. Bath, J.G. Gasser, J.L. Bretonnet, R. Bianchin et R. Kleim, J. Phys. Colloques 41 C8519-C8-523 (1980).

[18] L. Verlet, Phys. Rev. 159 (1967) p.98; Phys. Rev. 165 (1968) p.201. 
[19] L. D. Lucas, «Techniques de l'Ingénieur, Traité des Matériaux Métalliques », Formulaire M65, France.

[20] A. Witkowska, J. Rybicki, S. De Panfilis, A. Di Cicco, J. Non-Cryst. Solids 352 (2006) p.4351.

[21] O. Dreirach, R. Evans, H. Güntherodt and H.-U. Kunzi, J. Phys. F2 (1972) p.709.

[22] G. Mukhopadhyay, A. Jain and V. K. Ratti; Sol. St. Com. 13, (1973) p.623.

[23] A. Ben Abdellah, J. G. Gasser, A. Makradi, B. Grosdidier and J. Hugel, Phys. Rev. B 68 (2003) p.184204.

[24] E. Esposito, H. Ehrenreich and S. D. Gelatt, Phys. Rev. B 18 (1978) p.3913.

[25] J. R. Wilson, Met. Rev. 10 (1965) p.381.

[26] L. Sani L.E. Bove, C. Petrillo and F. Sacchetti, J. Non-Cryst. Solids 353 (2007) p.3139. 


\section{TABLE CAPTIONS}

Table 1. The characteristic parameters of radial distribution function, $g(r)$, of liquid bismuth; $g\left(r_{1}\right)$ and $r_{1}$ indicate the first peak value of $g(r)$ and the corresponding $r$ value; $g\left(r_{2}\right)$ and $r_{2}$ indicate the second peak value of $g(r)$ and the corresponding $r$ value.

Table 2: The characteristic parameters of structure factor, $S(q)$, of liquid Bi; $S\left(q_{1}\right)$ and $q_{1}$ indicate the first peak value of $S(q)$ and the corresponding $q$ value; $S\left(q_{2}\right)$ and $q_{2}$ indicate the second peak value of $S(q)$ and the corresponding $q$ value.

Table 3: Liquid bismuth experimental [15] (second column) and calculated electrical resistivity using 5 conduction electrons (third column) and using 3 conduction electrons (fourth column).

\section{FIGURES CAPTIONS}

Figure 1. Indlekofer [13] experimental (full line) and Hafner Jank [5] calculated (dashed line) Density Of State (DOS) of liquid bismuth.

Figure 2. Liquid bismuth effective potentials calculated at $T=300^{\circ} \mathrm{C}$ from ECP and OMP pseudo-potentials for different values of the valencies $Z$. Full line: ECP, $Z=3$. Dashed line: ECP, $Z=5$. Dotted line: for OMP, $Z=3$. Dashed dotted line: OMP, $Z=5$.

Figure 3. Liquid bismuth effective potentials calculated at $T=300^{\circ} \mathrm{C}$ from ECP pseudopotential with the valence $Z=5$ for both values of core radius full line: $R_{E C P}=0.529 \AA$ and 
Dashed line: $R_{E C P}=1.005 \AA$ and with the valence $Z=3$ for both values of core radius dotted line: $R_{E C P}=0.587 \AA$ and dashed-dotted line: $R_{E C P}=0.878 \AA$.

Figure 4. Liquid bismuth Waseda [16] experimental (circle) and computed pair correlation functions from the effective potentials calculated on figure 3 Full line: $R_{E C P}=0.529 \AA$. Dashed line: $R_{E C P}=1.005 \AA$. Dotted line: $R_{E C P}=0.587 \AA$ and dashed-dotted line: $R_{E C P}=0.878 \AA$.

Figure 5. Liquid bismuth effective potentials calculated at $T=300{ }^{\circ} \mathrm{C}$ with the valence $Z=5$ and from OMP pseudo-potential for different values of core radius $R_{O M P}$. Full line: $R_{\text {OMP }}=0.95 \AA$. Dashed line: $R_{\text {OMP }}=1.00 \AA$. Dotted line: $R_{\text {OMP }}=1.05 \AA$.

Figure 6. Liquid bismuth Waseda [16] experimental (circle) and computed pair correlation functions from the effective potentials calculated in figure 5. Full line: $R_{O M P}=0.95 \AA$. Dashed line: $R_{O M P}=1.00 \AA$. Dotted line: $R_{O M P}=1.05 \AA$.

Figure 7. Liquid bismuth Waseda [16] experimental structure factor (circle) and our calculated structure factors obtained by Fourier transform from our pair correlation functions. Full line: $R_{O M P}=0.95 \AA$. Dashed line: $R_{O M P}=1.00 \AA$. Dotted line: $R_{O M P}=1.05 \AA$.

Figure 8. Ion-ion effective potential for the liquid metal bismuth ((a): $Z=3, T=300^{\circ} \mathrm{C}$, (b): $Z=3, T=550{ }^{\circ} \mathrm{C}$ and (c) $Z=3, T=800^{\circ} \mathrm{C}$ ). 
Figure 9. Waseda [16] experimental (circle) and computed (full line) pair correlation functions for the liquid metal bismuth ((a): $Z=3, T=300^{\circ} \mathrm{C}$, (b) $Z=3, T=550^{\circ} \mathrm{C}$ and (c) $\left.Z=3, T=800^{\circ} \mathrm{C}\right)$

Figure 10. Waseda [16] experimental structure factor (circle) and calculated static structure factors (full fine) obtained by Fourier transform from our pair correlation function for the liquid metal bismuth ((a): $Z=3, T=300^{\circ} \mathrm{C}$, (b) $Z=3, T=550^{\circ} \mathrm{C}$ and (c) $Z=3, T=800^{\circ} \mathrm{C}$ ). 


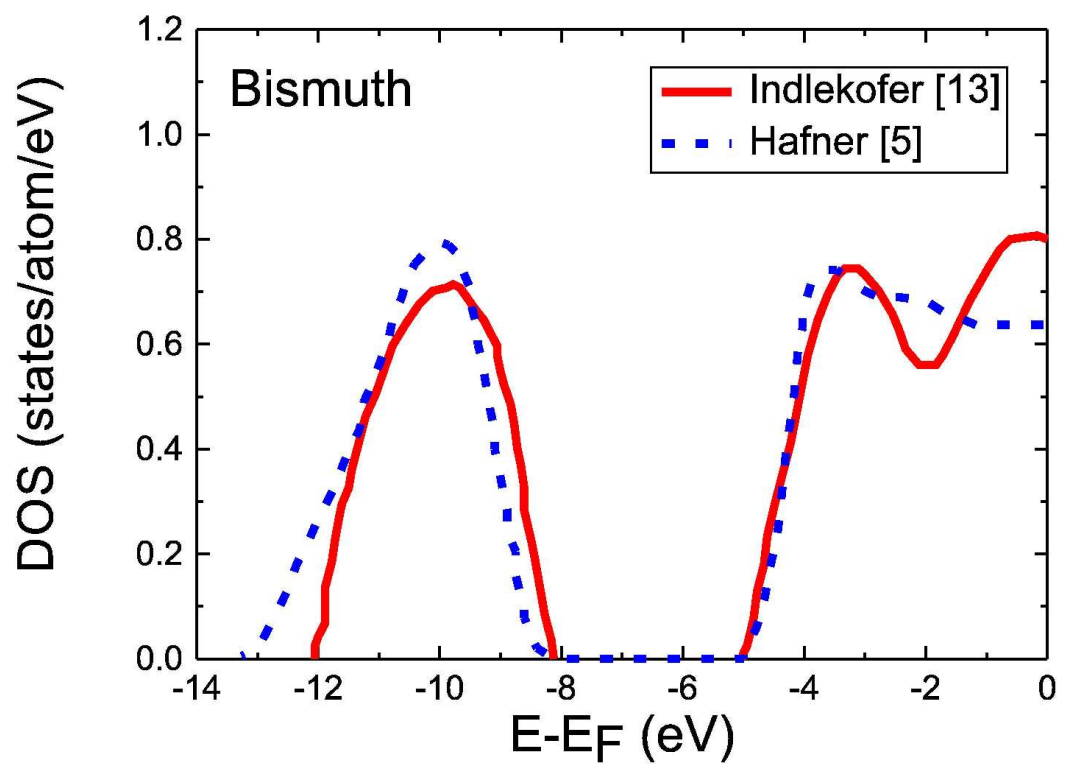

$149 \times 111 \mathrm{~mm}(600 \times 600 \mathrm{DPI})$ 


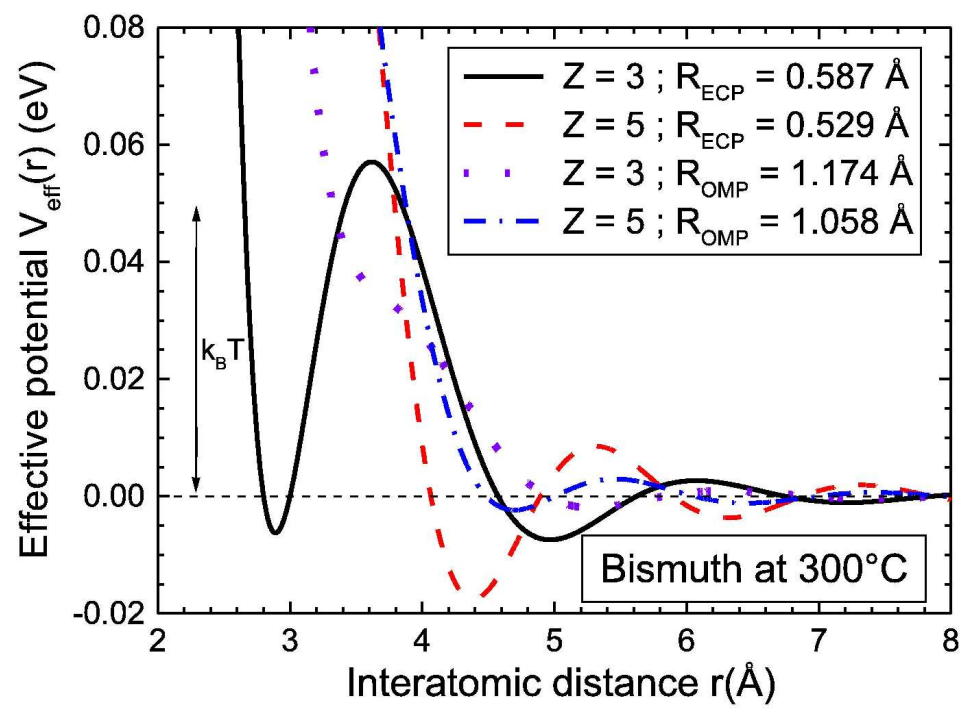

$160 \times 108 \mathrm{~mm}(600 \times 600 \mathrm{DPI})$ 


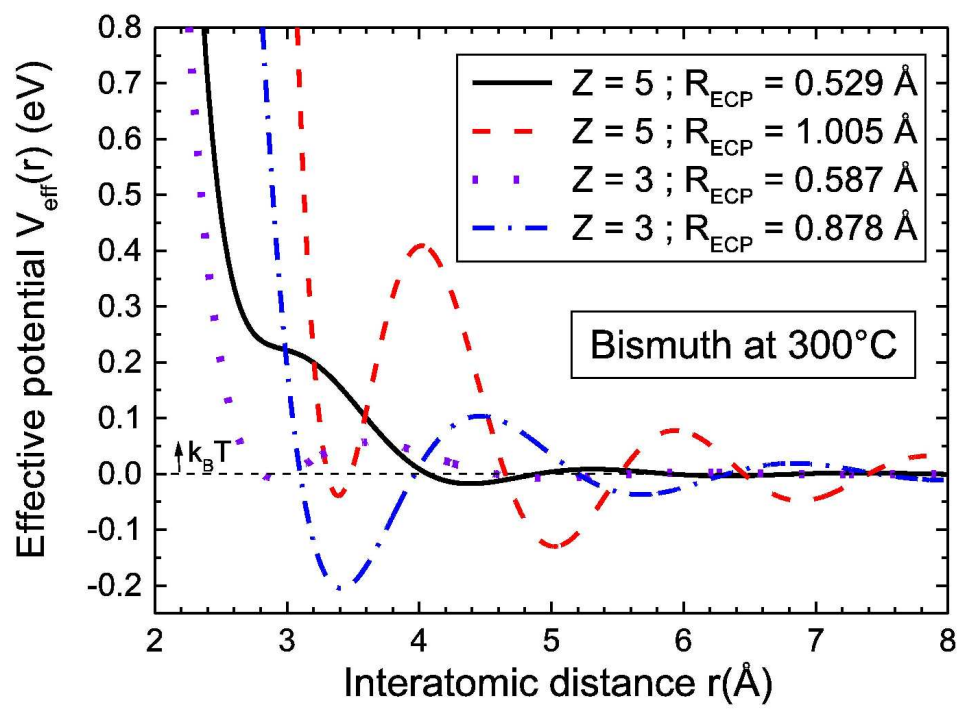

$160 \times 108 \mathrm{~mm}(600 \times 600 \mathrm{DPI})$ 


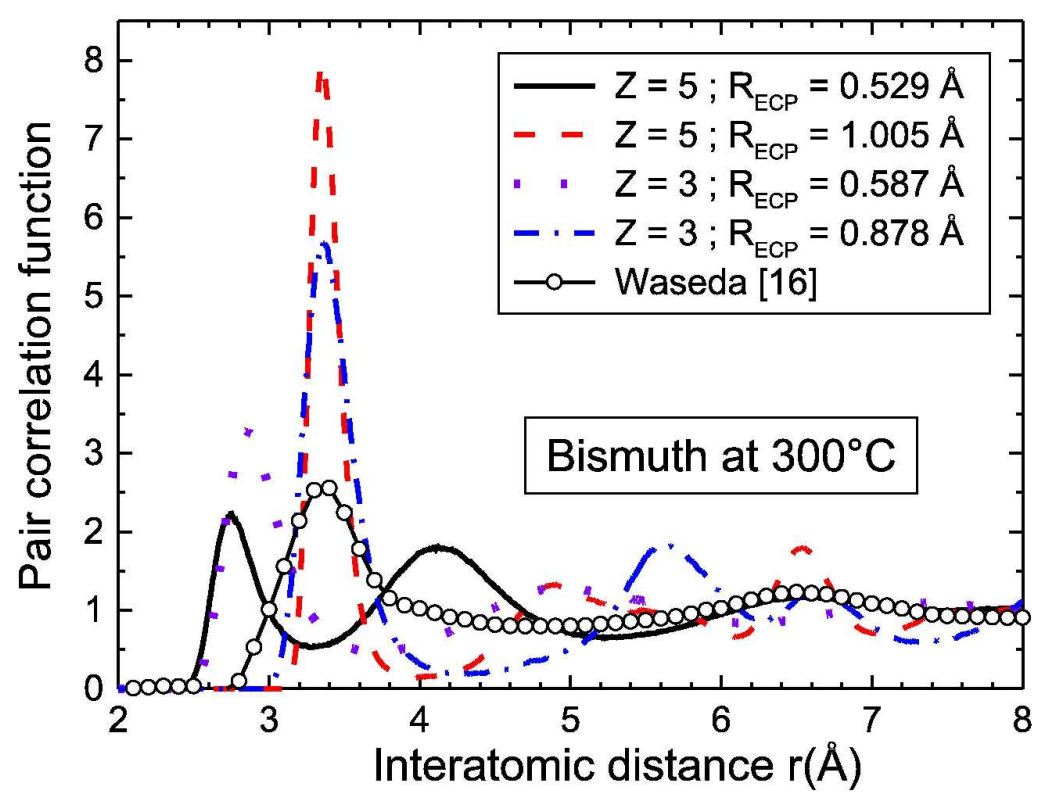

$141 \times 108 \mathrm{~mm}(600 \times 600 \mathrm{DPI})$ 


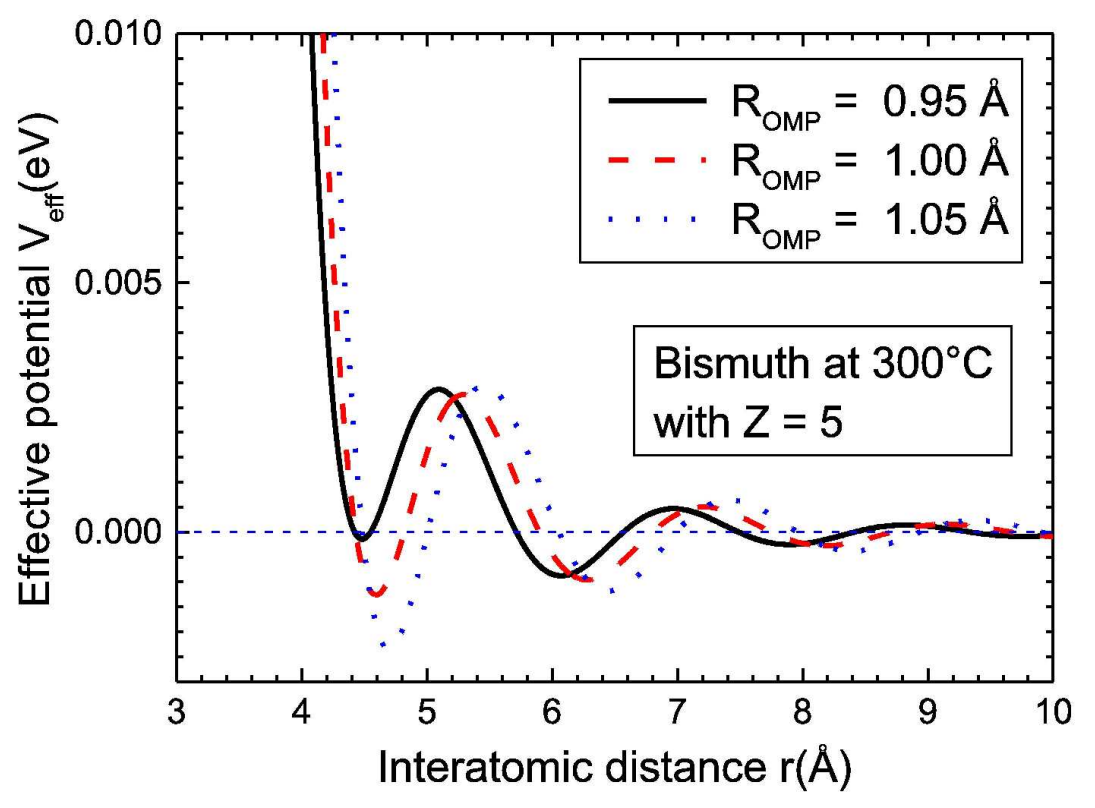

$145 \times 109 \mathrm{~mm}(600 \times 600 \mathrm{DPI})$ 
Figure 6

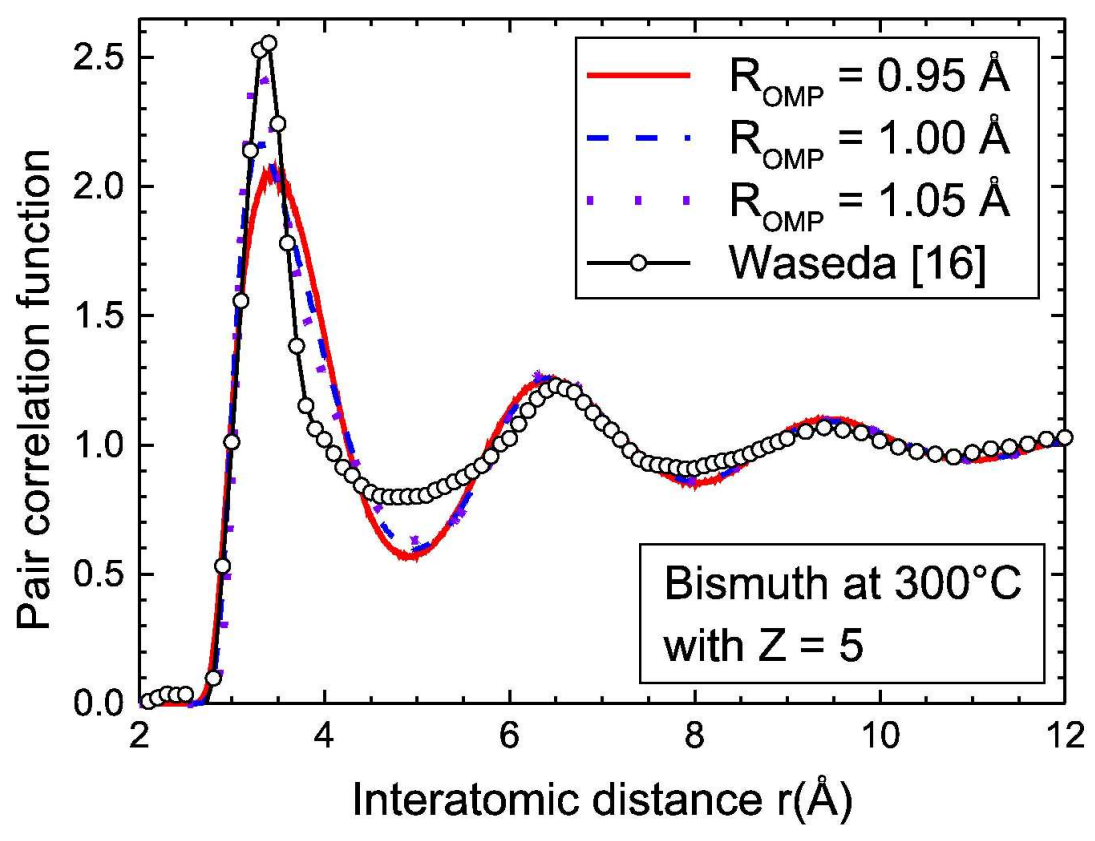

$137 \times 110 \mathrm{~mm}(600 \times 600 \mathrm{DPI})$ 


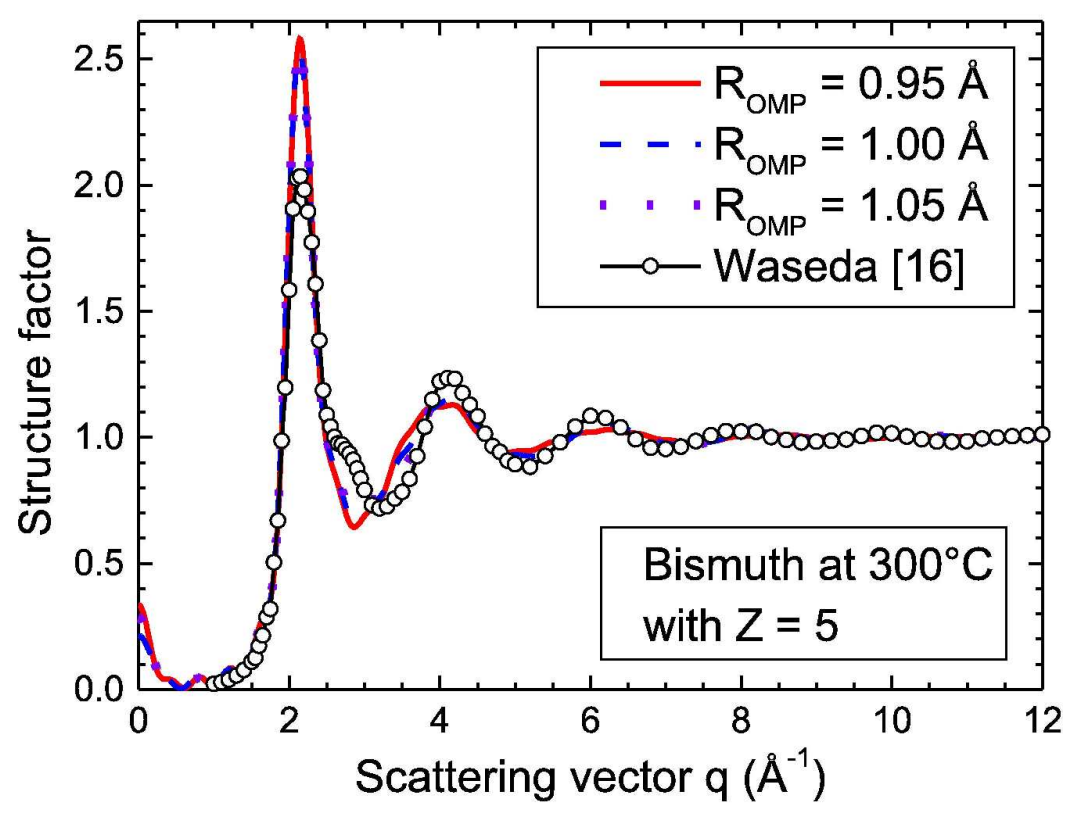

$141 \times 110 \mathrm{~mm}(600 \times 600 \mathrm{DPI})$ 
Figure 8

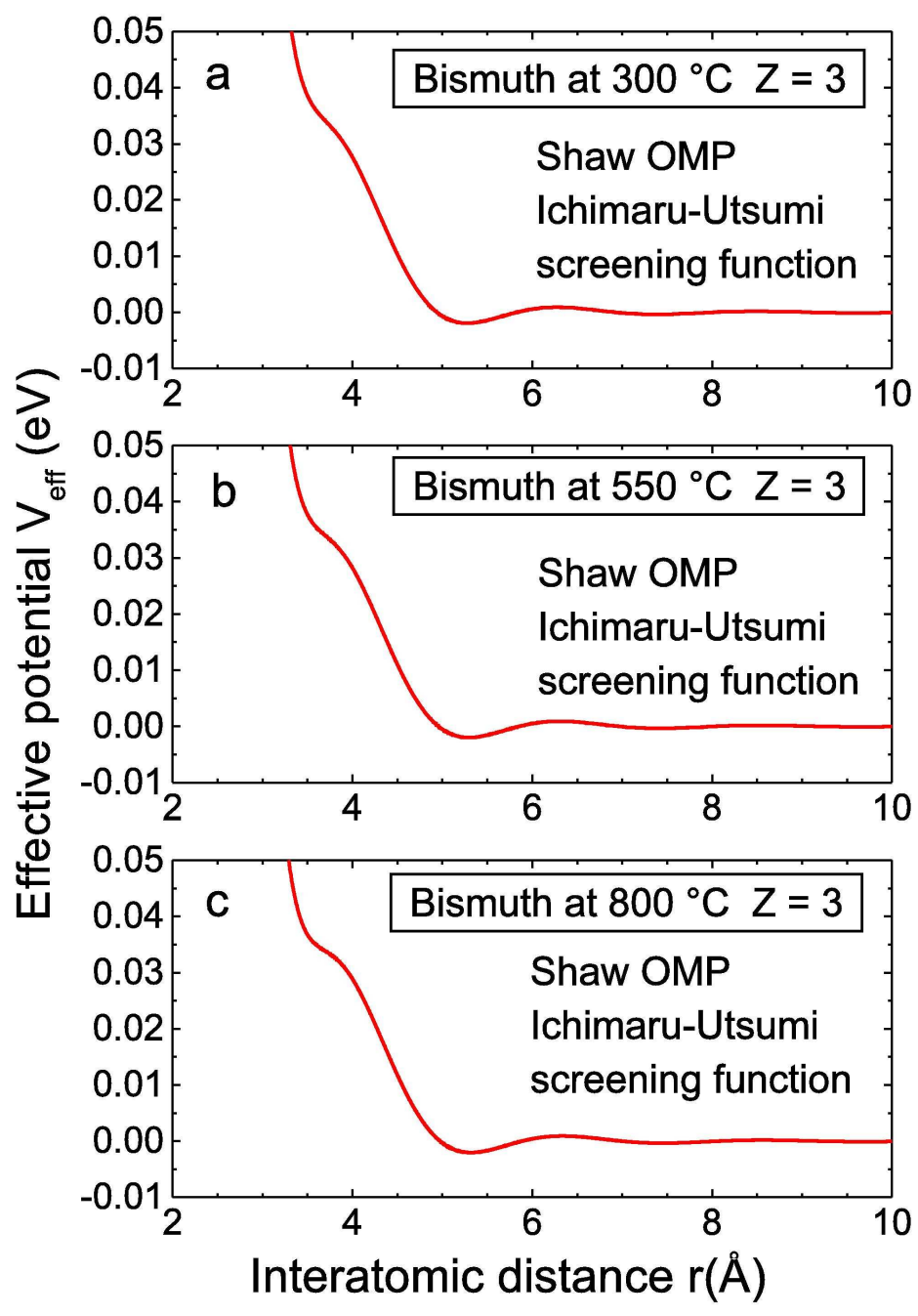

$99 \times 141 \mathrm{~mm}(600 \times 600 \mathrm{DPI})$ 
Figure 9

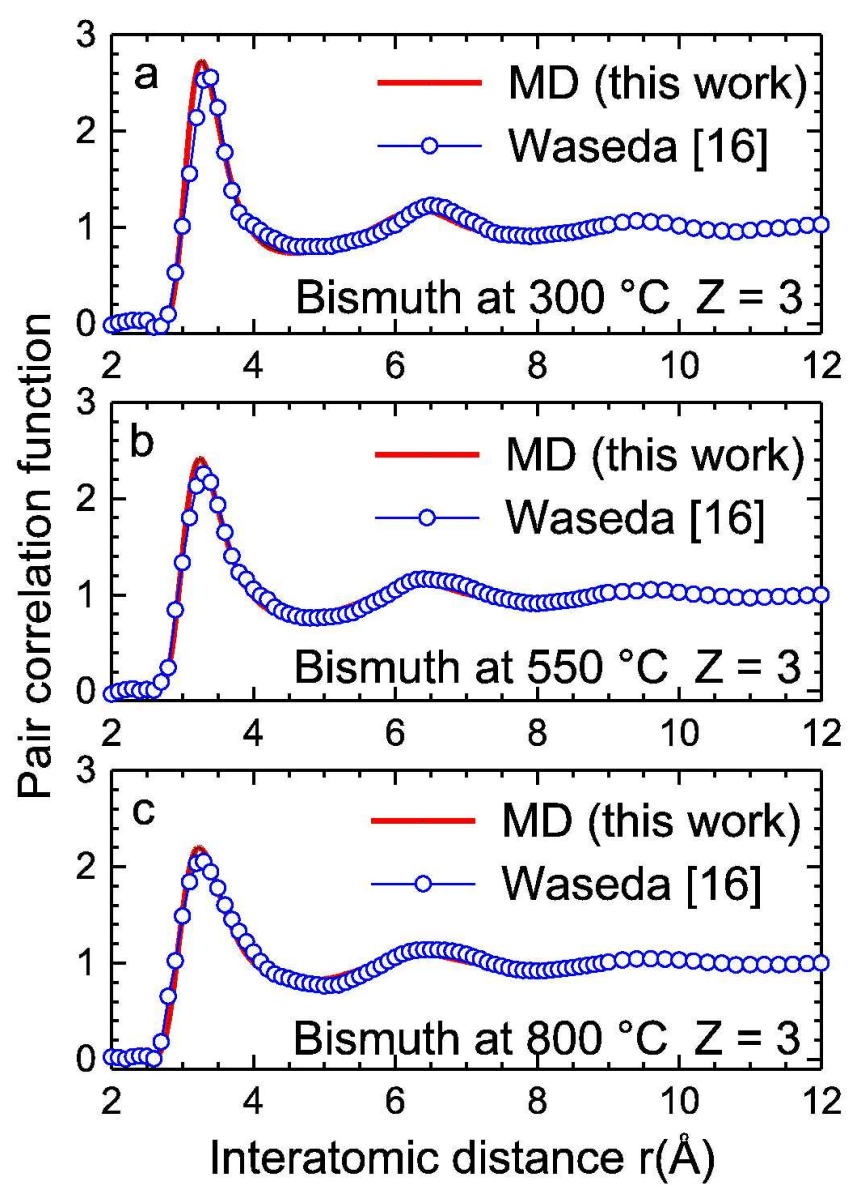

$116 \times 163 \mathrm{~mm}(600 \times 600 \mathrm{DPI})$ 
Figure 10

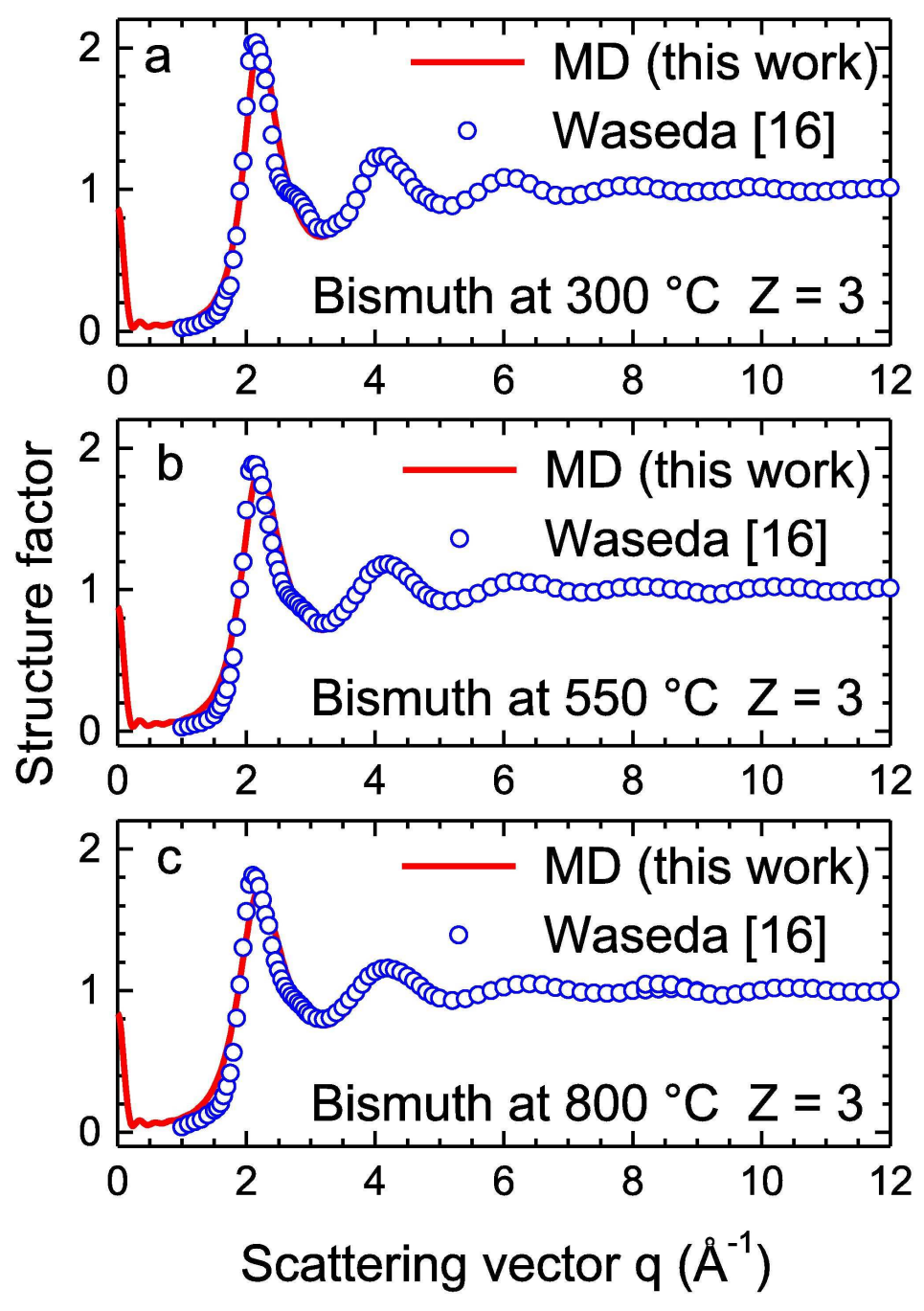

$101 \times 143 \mathrm{~mm}(600 \times 600 \mathrm{DPI})$ 
Table 1:

\begin{tabular}{|l|l|l|l|l|l|l|}
\hline$T\left({ }^{\circ} \mathrm{C}\right)$ & $q_{1}\left(\AA^{-1}\right)$ & $S_{1}(q)$ & $q_{2}\left(\AA^{-1}\right)$ & $S_{2}(q)$ & $q_{2} / q_{1}$ & $S\left(q_{2}\right) / S\left(q_{1}\right)$ \\
\hline 300 & 2.21 & 2.0576 & 4.16 & 1.2538 & 1.88 & 0.609 \\
\hline 550 & 2.21 & 1.8487 & 4.20 & 1.1981 & 1.90 & 0.648 \\
\hline 800 & 2.20 & 1.7130 & 4.22 & 1.1595 & 1.92 & 0.676 \\
\hline
\end{tabular}


Table 2:

\begin{tabular}{|l|l|l|l|l|l|l|l|}
\hline$T\left({ }^{\circ} \mathrm{C}\right)$ & $r_{1}(\AA)$ & $g\left(\mathrm{r}_{1}\right)$ & $r_{2}(\AA)$ & $g\left(\mathrm{r}_{2}\right)$ & $r_{2} / r_{1}$ & $g\left(\mathrm{r}_{2}\right) / g\left(\mathrm{r}_{1}\right)$ & $\begin{array}{c}\text { First } \\
\text { coordination } \\
\text { number }\end{array}$ \\
\hline 300 & 3.2709 & 2.7227 & 6.3265 & 1.1835 & 1.93 & 0.434 & 9.47 \\
\hline 550 & 3.2563 & 2.4147 & 6.3048 & 1.1390 & 1.93 & 0.471 & 8.99 \\
\hline 800 & 6.3050 & 2.1998 & 3.2356 & 1.1064 & 1.95 & 0.50 & 8.21 \\
\hline
\end{tabular}


Table 3:

\begin{tabular}{|c|c|c|c|}
\hline $\begin{array}{c}\text { Temperature } \\
T\left({ }^{\circ} \mathrm{C}\right)\end{array}$ & $\begin{array}{c}\text { Experimental } \\
\text { resistivity } \\
\rho(\mu \Omega \cdot \mathrm{cm})\end{array}$ & $\begin{array}{c}s+p \text { valence } \\
\text { Resistivity } \\
\rho(\mu \Omega \cdot \mathrm{cm})\end{array}$ & $\begin{array}{c}p \text { valence } \\
\text { Resistivity } \\
\rho(\mu \Omega \cdot \mathrm{cm})\end{array}$ \\
\hline 300 & {$[15]$} & $Z=5$ & $Z=3$ \\
& 128.54 & 57.77 & 143.65 \\
\hline 550 & {$[15]$} & $Z=5$ & $Z=3$ \\
& 141.78 & 59.59 & 145.65 \\
\hline 800 & {$[15]$} & $Z=5$ & $Z=3$ \\
& 155.00 & 70.94 & 197.60 \\
\hline
\end{tabular}

